\title{
Mutagen formation potential of river water and removal of mutagen precursor by activated carbon
}

\author{
H. Takanashi ${ }^{1}$, T. Nakajima ${ }^{1}$, A. Ohki ${ }^{1}$, S. Kokubu ${ }^{2}$, M. Hirata ${ }^{2}$ \\ \& $\mathrm{T}$. Hano ${ }^{2}$ \\ ${ }^{I}$ Department of Bioengineering, Kagoshima University, Japan \\ ${ }^{2}$ Department of Applied Chemistry, Oita University, Japan
}

\begin{abstract}
The mutagen formation potential (MFP) was measured for the river water, the raw sewage and the effluent of the sewage works by means of the Ames Salmonella mutagenicity assay. MFP of the river water samples ranged from 1,200 to 22,000 net revertant colonies per liter of sample water. MFP of the effluent was from 5,300 to 22,000 net revertant colonies per liter. This survey showed that the removal percentages of dissolved organic carbon (DOC) at the sewage works were $62.0-79.3 \%$. It also showed that MFP reductions at the same sewage works were $37.5-62.9 \%$. There was no significant correlation observed between MFP and organic matter concentration for the river water samples. However, a weak correlation between ammonia nitrogen and MFP was observed for the same river water samples. These results indicate that MFP cannot be accurately estimated by use of the conventional water quality indices. When the samples contain a high concentration of ammonia nitrogen, it is difficult to control the amount of free chlorine dosages stoichiometrically, possibly resulting in the overdose of chlorine. An overdose of chlorine may cause further formation of mutagens. The isotherm of mutagen precursors adsorption onto the activated carbon (OL 20x50), which was purchased from Calgon Mitsubishi Chemical Corporation, was studied using river water in order to explore the means of removing mutagen precursors from raw water used for the water supply. The adsorption isotherm attained fit well into the Freundlich model.
\end{abstract}

Keywords: Ames Salmonella assay, chlorination, mutagenicity, mutagen formation potential, river water, sewage, activated carbon, adsorption isotherm. 


\section{Introduction}

More than two decades have passed since mutagens were found in tap water. A large number of other hazardous chemicals have also been identified [1]. But no major mutagens that can significantly explain the total mutagenic activity in tap water have been identified yet. The results of mutagenicity tests conducted on the tap water demonstrate that the tap water contains various unidentified mutagens, which are potentially hazardous to human beings.

A number of studies on the mutagenicity in tap water have been reported in various countries. For example, the authors [2] have reported that extremely high mutagenicity, 9,200 net revertant colonies per liter of sample water (hereafter referred to as net rev./L), was identified in some tap water in Japan from the study using the Ames mutagenicity assay with Salmonella typhimurium TA100 without exogenous activation by S9 mix (TA100-S9). The level of mutagenicity observed in the tested samples was more than 19 times of that of clean tap water samples in Japan.

In order to elucidate the causes of such high mutagenicity in the tap water samples, investigations were conducted on the mutagenicity of the surface water [3] and the mutagenicity changes occurring in water purification processes [4]. The results were; the mutagenicity of the surface water was extremely low compared to that of the tap water, and the mutagenicity had increased dramatically by chlorination during the water purification processes. These findings indicate that almost all mutagens in tap water are produced through the chemical reactions between several precursors and chlorine. This can be supported by the facts that humic substances present in surface water react with chlorine, resulting in the production of numerous mutagenic by-products (DBPs) [5]. However, the chlorination of water is indispensable when providing safe tap water from the sources bearing microbes for diseases of the digestive system. Therefore it is essential that the river water be prevented from being polluted by mutagen precursors.

The authors have proposed a new water quality index for the mutagen formation potential in chlorination (MFP), which is defined as mutagenicity of the sample chlorinated under the same conditions as in the water purification processes [6]. By applying the new index, we have investigated MFP for 65 discharged wastewaters, which meet the Japanese wastewater quality standards, and accordingly studied the possibility of mutagenic contamination of the river waters by these wastewaters. From the results, we reported that MFP of the sewage effluent showed an intermediate level among the other wastewaters tested, but MFP load of the sewage effluent was considerably larger than those of the others because the amount of the sewage effluent was generally larger [7].

This study was conducted by assessing MFP of forty-one samples collected from rivers in the Kyushu region, which are located in the southern part of Japan. Each tested river supplies water to its nearby city. The test results were compared with MFP of the effluent from the sewage works. Furthermore, the adsorption isotherm of mutagen precursors onto an activated carbon was studied for the purpose of removing mutagen precursors from the river waters. 


\section{Materials and methods}

\subsection{Samples}

Forty-one river water samples were collected from July 2002 to December 2003. The experiments were conducted within two days after the sampling. The sampling sites were chosen from class A rivers whose raw waters were taken into the water works. The locations of the sampling sites are illustrated in figure 1 . The sampling was made only when the weather was fair or cloudy during the past three days.

The untreated (raw) sewage samples were collected at the primary settling reservoirs of four different terminal treatment plants used for the separate-type sewer systems located in the Kyushu region. The treated samples (effluent) were collected at the aeration tanks in eight different terminal treatment plants used for the separate-type sewer system, including the above four plants.

Mutagenicity was not detected in the tests for any water samples before chlorination (data not shown).

Dissolved organic carbon (DOC) concentrations were measured by means of an analyzer, model TOC-5000A (Shimadzu Corporation, Ltd.), for the water samples screened with a $0.45 \mu \mathrm{m}$-pore membran filter. $\mathrm{A}_{260}\left(\mathrm{E}_{260}\right)$ was determined as the absorbance at a wavelength of $260 \mathrm{~nm}$. Ammonia nitrogen concentrations were determined by colorimetry, using the indophenol blue method or Nessler's method.

The activated carbon (OL 20x50) was purchased from Calgon Mitsubishi Chemical Corporation, Japan. It was boiled in pure water before use to remove air from the pores of the activated carbon.

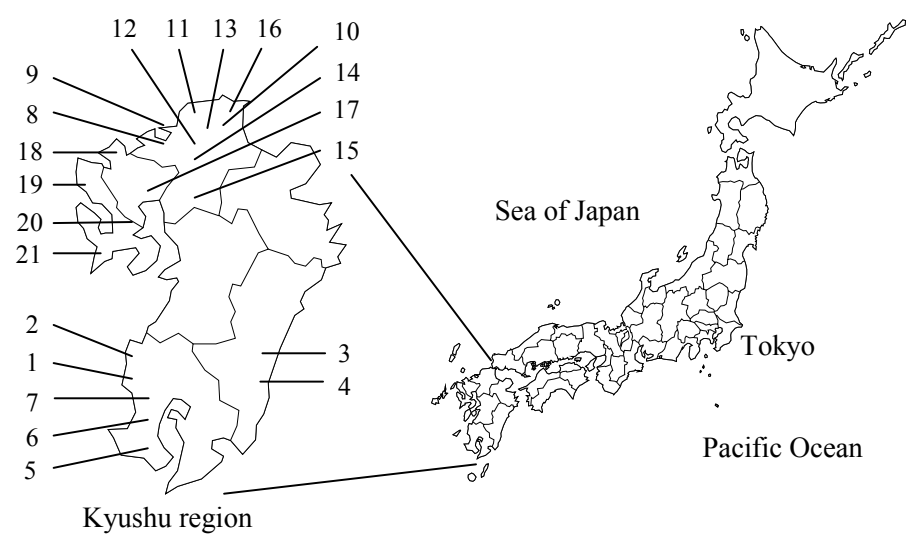

Figure 1: Location map of sampling site.

\subsection{Sample preparation for Ames assay}

Before measuring MFP, the water samples were chlorinated according to the procedure [6] illustrated in figure 2. The mutagens produced through the 
chlorination were concentrated 1,000 times by the adsorption-desorption method (a solid phase extraction method) [6]. This method can recover more than $90 \%$ of the mutagens present from various water samples within an hour. The concentration procedure is illustrated in figure 3 .

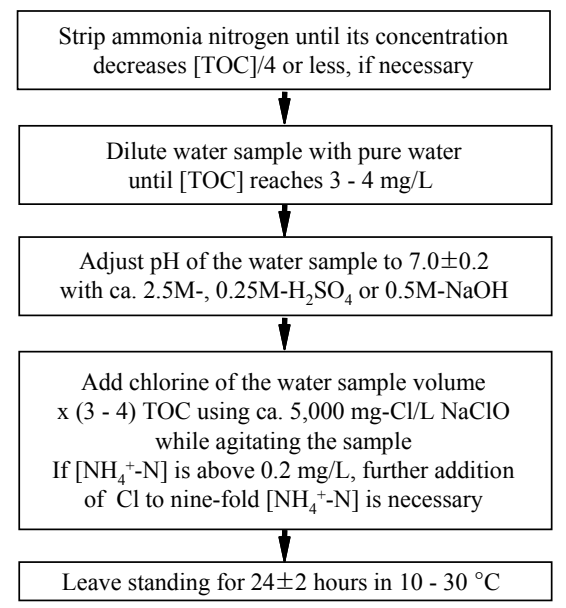

Figure 2: $\quad$ Chlorination procedure for measuring MFP.

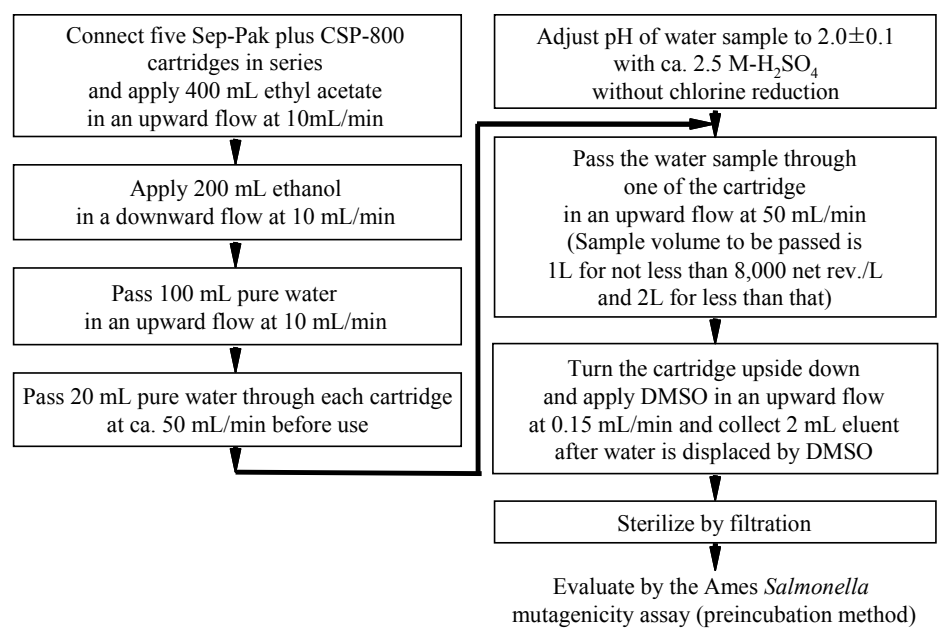

Figure 3: $\quad$ Sample preparation procedure for Ames assay.

\subsection{Ames Salmonella mutagenicity assay}

The mutagenicity of the concentrated samples was examined by the Ames Salmonella mutagenicity assay [8] after sterilizing the eluate by filtration through a $0.45 \mu \mathrm{m}$-membrane filter. The preincubation method [9] using 
Salmonella typhimurium TA100 without exogenous activation by S9 mix (TA100-S9) was employed. These conditions, TA100-S9, have been most widely used for testing the chlorinated water samples because many mutagens show direct mutagenicity and cause base-pair substitutions in genes [10].

Dose-response tests were carried out using three dose-steps and one negative control. Duplicate and quadruplet plates were used for each the dose-step and the negative control step, respectively. Positive control tests were also carried out concurrently with a DMSO solution of 4-nitroquinoline-1-oxide (4NQO) in order to confirm the stability of the activity of the strain. MFP of the samples was evaluated as net rev./L, which was calculated from the slope of dose-response lines. All of the water samples showed dose-response lines with good linearity (data not shown).

\section{Results and discussion}

\subsection{Mutagen formation potential of the samples}

DOC and MFP of the water samples from the raw sewage, the effluents of the sewage works and the river waster were plotted in figure 4. DOC of each was 12.2-28.0, 4.7-16.5 and 1.2-3.3 mg/L, respectively. MFP of each was 12,00022,300, 5,300-22,200 and 1,200-22,000 net rev./L, respectively. The maximum MFP of the river water samples was 22 times the mean mutagenicity of the tap water in Japan, which was obtained from the investigation on the tap water samples of 23 prefectures from 1992 to 1993 [2].

DOC removal and MFP reduction in the sewage works were 62.0-79.3 and 37.5-62.9\%, respectively. There was no case where MFP increased through the water treatment processes in the sewage works. There is a report [12] that oxidation processes, such as ozone treatment, have a possibility to increase mutagenicity due to the formation of glyoxal derivatives. However, only the conventional activated treatment, not the ozone treatment, was applied at the sewage works that were used in this study.

Two-sample $t$-tests were performed to study the difference between MFP of the river waters and the effluents. The level of significance was calculated to be 0.00385 , which means the difference of MFP is significant. It is considered that the sewage discharges are diluted in the rivers, thereby decreasing mutagen precursor concentration in the rivers. The ratios of sewage discharge to the river water flow in this study are presently unknown because of lack of data regarding the river water flow. Because the mixing conditions of the rivers are also unknown, a detailed discussion was unable to be held in this study. It is presumed, however, the ratio is much smaller than $71.0 \%$ because the ratio on the Sumida River, which is one of the most urbanized rivers running through metropolitan Tokyo, was reported to be $71.0 \%$ at Ryogoku Bridge near the estuary [13].

The statistic study results indicate that MFP of the river waters and the effluents were significantly different as described above. But the most remarkable point in figure 4 is that the maximum values of MFP of the river 
waters and the effluents were comparable. This is probably because the river waters are polluted by other pollution sources than sewage discharges. Further investigation needs to be done to find the other sources.

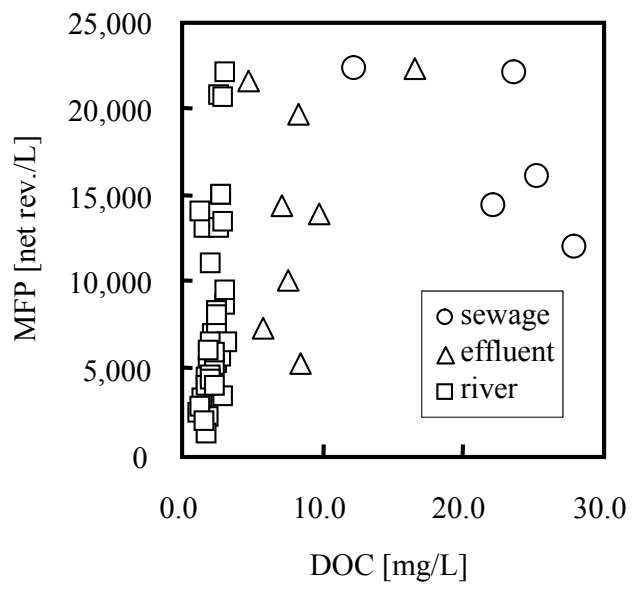

Figure 4: Correlation between DOC and MFP.

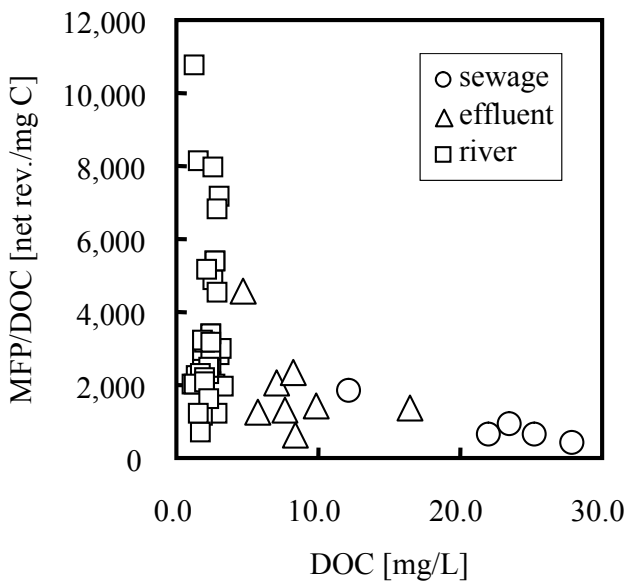

Figure 5: $\quad$ MFP/DOC of various kinds of water.

The second point in figure 4 is that MFP reduction is low compared with DOC removal. High DOC removal in the sewage works indicates that the sewage treatment processes are functioning correctly. This means that biodegradability of mutagen precursors in the sewage is low. It is expected that advanced sewage treatments, such as activated carbon treatment or UV treatment, will be studied in the near future for the purpose of removing mutagen precursors. 


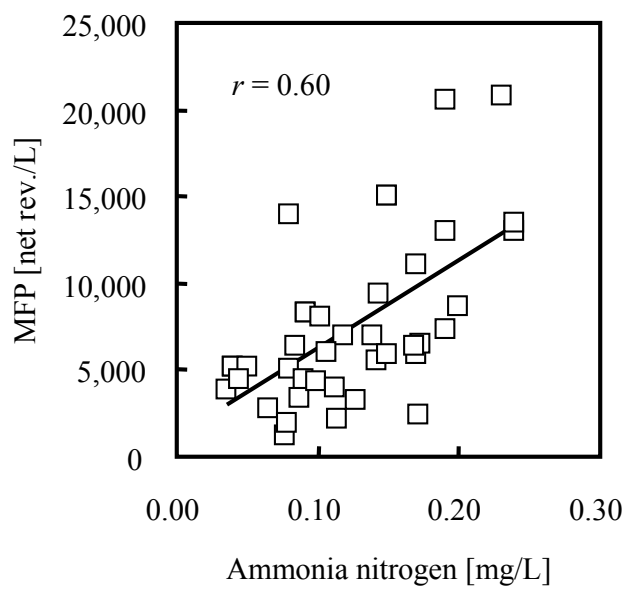

Figure 6: Correlation between ammonia nitrogen and MFP.

MFP per unit weight of DOC (MFP/DOC), which is a relative value, was quite different from the river waters and the others, as shown in figure 5. These results support the above presumption, which the river waters are polluted by sources other than sewage discharges.

\subsection{Correlation between MFP and conventional water quality indices}

Correlations between MFP and DOC, $\mathrm{A}_{260}$ and ammonia nitrogen concentration were investigated for the river waters. The correlations for the raw sewage waters and the effluents were not studied because the number of samples was insufficient. No ammonia nitrogen was removed from all the river water samples because the ammonia nitrogen concentration in the samples was less than [TOC]/4. The correlations between MFP and DOC, MFP and ammonia nitrogen concentration were shown in figures 4 and 6 . The Pearson correlation coefficients between MFP and DOC, MFP and $\mathrm{A}_{260}$, MFP and ammonia nitrogen concentration were $0.46,0.39$ and 0.60 , respectively.

No significant correlation between MFP and the organic matter concentration was observed. These results show that MFP cannot be accurately estimated from the conventional water quality indices, DOC and $\mathrm{A}_{260}$. Therefore MFP should be measured as an original index.

However, a weak correlation was observed between MFP and ammonia nitrogen concentration. It is well known that ammonia nitrogen consumes free chlorine and decreases the formation of trihalomethanes (THMs). This is because the reaction of chlorine with ammonia nitrogen to form chloramines is very fast compared to the reaction of chlorine with natural organic matters, and also because the chloramines produced do not form THMs [14]. As the major mutagens in chlorinated water samples are considered to be chlorination byproducts, ammonia nitrogen is considered to decrease mutagens formation. Nevertheless, MFP as well as ammonia nitrogen concentration increased, as 
shown in Figure 6. This is probably because additional chlorine was applied to the samples which contain ammonia nitrogen, for the purpose of sufficient formation of chloramines during MFP measuring procedure shown in figure 2 . Because it is difficult to control the dosage of free chlorine stoichiometrically when the samples contain a high concentration of ammonia nitrogen, there is a possibility of an overdose of free chlorine. An overdose of chlorine potentially causes further formation of mutagenicity. Overdoses of chlorine to raw waters that contain high concentration of ammonia nitrogen are occurring in actual water purification processes.

\subsection{Adsorption isotherm of mutagen precursor onto activated carbon}

Adsorption isotherms of natural organic matters and mutagen precursors onto activated carbon were studied in order to remove mutagen precursors from the river waters at $298 \mathrm{~K}$ and $\mathrm{pH} 7.0$. Based on the batch experimental results, $61 \%$ of MFP reduction and $65 \%$ of DOC removal were achieved for river waters under the condition of $4.5 \mathrm{~g} / \mathrm{L}$ of activated carbon concentration (data not shown). The adsorption isotherms were studied and they fit well into the Freundlich model. The adsorption isotherm on mutagen precursors is shown in figure 7.

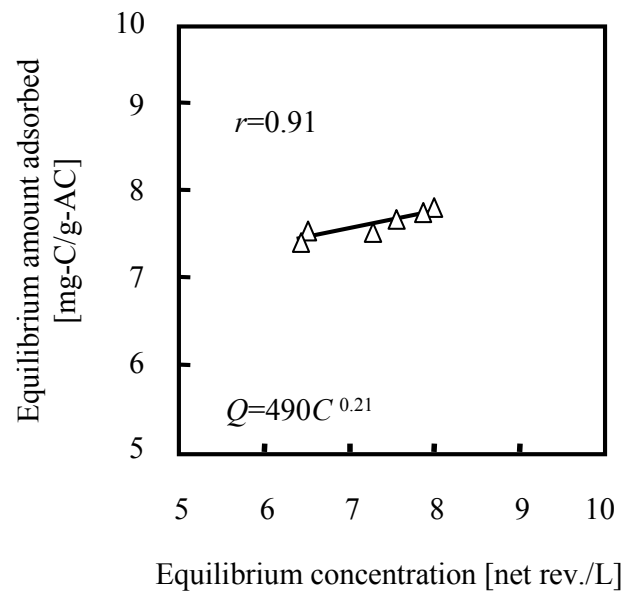

Figure 7: Adsorption isotherm of mutagen precursors in river water onto activated carbon, OL $20 \times 50$.

A breakthrough curve of mutagen was simulated based upon the mass transfer zone method according to the equilibrium study results and under the following assumptions; the rate-determining stage of adsorption is that of mass transfer in intraparticle, the effective intraparticle diffusion coefficient is $2 \times 10^{-12} \mathrm{~cm}^{2} / \mathrm{s}$, MFP of column inlet is 5,300 net rev./L, the particle diameter of the activated carbon packed is $0.8 \mathrm{~mm}$, the temperature is $298 \mathrm{~K}$ and the $\mathrm{pH}$ is 7.0 . In order to maintain MFP of the column outlet within 3,000 net rev./L for 30 days of 
operation period, 220 min of empty bed contact time was needed. The results indicate that activated adsorption processes are impractical for reducing MFP in water purification processes.

\section{Conclusions}

MFP of forty-one river water samples, four raw (untreated) sewage samples and eight effluent samples from sewage works were measured and they were 12,00022,300, 5,300-22,200 and 1,200-22,000 net rev./L, respectively. The maximum MFP of river waters and the effluent from sewage works were comparable. River waters are considered to be polluted by other pollution sources than sewage discharges. Biodegradability of mutagen precursors in sewage is considered to be low.

MFP cannot be accurately estimated by use of the conventional water quality indices because no significant correlation between MFP and organic matter concentration was observed for the river water samples. However, a weak correlation was observed between MFP and ammonia nitrogen concentration. This is probably because additional chlorine was applied to the samples which contain ammonia nitrogen, for the purpose of sufficient formation of chloramines during MFP measuring procedure.

The adsorption isotherm of mutagen precursors in a river water sample onto an activated carbon, OL 20x50 purchased from Calgon Mitsubishi Chemical Corporation fit well into the Freundlich model as $Q=490 C^{0.21}$. Due to poor adsorption capacity of mutagen precursors, activated adsorption processes are impractical for reducing MFP in water purification processes.

\section{Acknowledgements}

The authors would like to acknowledge the funding for the studies from Ministry of Education, Science, Sports and Culture, Japan (Grant-In-Aid for Young Scientists, No. 15710064.). The authors are grateful to the students, Mr. Hisashi Wakikuromaru, Hitoshi Kajiya, Kaori Miyahara and Mieko Akama for their excellent experiments.

\section{References}

[1] World Health Organization, Revision of the WHO Guidelines for Drinking water Quality, WHO, Geneva, Switzerland, 1996.

[2] Urano, K., Okabe, F., Takanashi, H., and Fujie, K., Ames Mutagenicity of Tap water III. Analysis of Mutagenicity Level of Tap Water in Japan, J. Japan Society on Water Environment, 18, 1001-1011, 1995 (in Japanese).

[3] Aleem, A. and Malik, A., Genotoxicity of the Yamuna River Water at Okhla (Delhi), India, Ecotoxicology and Environmental Safety, 61, 404412, 2005. 
[4] Zani, C., Feretti, D., Buschini, A., Poli, P., Rossi, C., Guzzella, L., Caterino, F.D. and Monarca, S., Toxicity and Genotoxicity of Surface Water before and after Various Potabilization Steps, Mutation Research, 587, 26-37, 2005.

[5] Rook, J.J., Formation of Haloforms during Chlorination of Natural Waters, Water Treatment Examination, 23, 234-243, 1974.

[6] Takanashi, H., Urano, K., Hirata, M., Hano, T. and Ohgaki, S., Method for Measuring Mutagen Formation Potential (MFP) on Chlorination as a New Water Quality Index, Water Research, 35, 1627-1634, 2001.

[7] Takanashi, H., Urano, K. and Ohgaki, S., Analysis of Mutagen Formation Potential on Chlorination of Wastewater in Japan, J. Japan Society on Water Environment, 23, 352-359, 2000 (in Japanese).

[8] Ministry of Labour Japan, Mutagenicity Assay for the Occupational Safety and Health Act. Test Guideline and GLP, Tokyo Occupational Health Service Centre, pp173, 1991 (in Japanese).

[9] Yahagi, T., Degawa, M., Seino, Y., Matsushima, T., Nagao, M., Sugimura, T. and Hashimoto, Y., Mutagenicity of Carcinogenic Azo Dyes and Their Derivatives, Cancer Letter, 1, 91-96. 1975.

[10] Monarca, S., Ziglio, G., Pasquini, R., Beltramelli, G., Feretti, D., Donato, F., Nardi, G., Gervasoni, M., Micheli, F., Dalmiglio, A., Moretti, M., Mutagenicity of Drinking Water Obtained by Different Treatment Procedures from Two Northern Italian lakes, International J. Environmental Health Research, 2, 192-200, 1992.

[11] Takanashi, H. and Urano, K., Statistical Procedures for Estimating the Detection Limit and Determination Limit of the Ames Salmonella Mutagenicity Assay, Science of The Total Environment, 221, 31-42, 1998.

[12] Ueno, H., Nakamuro, K. and Sayato, Y., Glyoxal Derivatives as Ozonation Products: The Biodegradation, DNA Lesion in Rat Hepatocytes and the Occurrence in Water Purification Process, J. Japan Society on Water Environment, 18, 961-968, 1995 (in Japanese).

[13] Bureau of sewerage, Tokyo metropolitan government, http://www.gesui.metrotokyo.jp/k.anko/newst/154/n154_se.htm

[14] Amy, G. L., Chadik, P. A., King, P. H. and Cooper, W. J., Chlorine utilization during trihalomethane formation in the presence of ammonia and bromide, Environmental Science and Technology, 18(10), 781-786, 1984. 